\title{
Usando regras de associação para a identificação de falhas conceituais
}

\author{
Rodrigo Ruy Boguski ${ }^{1}$, Davidson Cury ${ }^{1}$ \\ ${ }^{1}$ Departamento de Informática - Universidade Federal do Espírito Santo (UFES) \\ Vitória - ES - Brasil \\ \{rodrigoboguski, dedecury\}@gmail.com
}

\begin{abstract}
Offer adapted teaching to the cognitive profile of the apprentice is a task that still unresolved in your completeness. In this paper is presented a strategy for getting the concepts not assimilated by a group of students in a learning event, using for this, association rules of data mining applied to conceptual maps.
\end{abstract}

Resumo. Oferecer ensino adaptado ao perfil cognitivo do aprendiz é uma tarefa ainda não resolvida em sua completude. Neste artigo é apresentada uma estratégia para obtenção dos conceitos não assimilados por um grupo de estudantes num evento de aprendizagem, utilizando para isto, regras de associação da mineração de dados aplicadas à mapas conceituais.

\section{Introdução}

Oferecer ensino adaptado ao perfil cognitivo do aprendiz é uma tarefa ainda não resolvida em sua completude, apesar de diferentes técnicas já haverem sido utilizadas. Visando preencher essa lacuna, utilizou-se como técnica as regras de associação da mineração de dados, adaptada ao contexto de mapas conceituais para obtenção do conjunto de conceitos não assimilados por um grupo de aprendizes. A intenção é que, posteriormente, esses conceitos sejam trabalhados de modo a nivelar o aprendizado coletivo, sem comprometer ou prejudicar a particularidade de cada aluno.

A mineração de dados é o processo de análise de conjuntos de dados que podem ser originados de diferentes fontes e tem por objetivo a descoberta de padrões interessantes que possam representar informações úteis, utilizando para isso diferentes técnicas. Ela não é um conceito essencialmente novo, entretanto, devido ao avanço da tecnologia relacionada ao processamento de informação, tornou-se ainda mais evidente nas últimas décadas pela diversidade de sua aplicação em atividades diárias. Esse conceito, pelo fato de ser interdisciplinar e não se restringir a uma única área, está presente em diversos contextos com diferentes aplicações.

Segundo [Han, Kamber \& Pei 2011], a mineração de dados é parte do processo de descoberta de conhecimento que contempla uma sequência iterativa dos passos de limpeza de dados, integração de dados, seleção de dados, transformação de dados, a própria mineração de dados, avaliação de padrões e apresentação do conhecimento. Nesse contexto, dispõe-se de algumas técnicas, [Han, Kamber \& Pei 2011] e [Amo 2004] que podem ser usadas de acordo com a finalidade, tais como associações, predições, regressões, clusterizações e suas variações. 
VII Congresso Brasileiro de Informática na Educação (CBIE 2018)

Anais do XXIX Simpósio Brasileiro de Informática na Educação (SBIE 2018)

Nosso objetivo é explicitar como os conceitos se relacionam e são percebidos por aprendizes em geral. Para isso, está sendo desenvolvida uma ferramenta que utiliza as regras de associação para operar em nosso projeto X [Cury, Perin \& Jr. 2014] em conjunção com outras ferramentas desse mesmo projeto.

Sobre os mapas conceituais pode-se dizer que são ferramentas gráficas para representar e organizar o conhecimento em duas dimensões, utilizando conceitos distribuídos de tal forma que as relações estabelecidas entre eles sejam evidentes [Novak \& Gowin 1984]. Eles têm sido utilizados nas mais diversas atividades pedagógicas que compõem o ensino-aprendizagem. Tanto as regras de associação, como os mapas serão apresentados sumariamente nas seções seguintes.

Este trabalho está organizado da seguinte forma: na seção 2 são apresentadas as regras de associação, na seção 3 a identificação de falhas conceituais e na seção 4 as considerações finais.

\section{Regras de Associação}

Suponha que você seja um professor de uma universidade e esteja interessado em conhecer os hábitos de estudo de seus alunos acerca de uma disciplina ou assunto como, por exemplo, quais conteúdos os alunos costumam acessar conjuntamente a cada vez que se dispõem a estudar certo assunto, ou seja, quais são as regras existentes de associação quando relacionam assuntos e conceitos entre si e a outros conceitos.

Conhecer a resposta para essa questão pode ser útil, assim você poderá planejar melhor o conteúdo em termos de objetos de aprendizagem (OA) [Flôres, Tarouco \& Reategui 2009], o relacionamento entre eles, assim como promover artifícios de incentivos a certos conteúdos, além de organizar melhor sua sequenciação e apresentação numa disciplina, colocando próximos os itens frequentemente acessados em conjunto a fim de encorajar os alunos a estudar tais lições e conceitos, maximizando o aprendizado. Para o desenvolvimento dessa questão é essencial que se conheça os conceitos de transação, itemset, suporte e confiança.

\subsection{Transação, itemset, suporte e confiança}

Considere que uma transação seja o registro de elementos associados pelo aluno em um evento de aprendizagem. É chamado de itemset cada conjunto de itens acessados pelo aluno numa única transação, assim, um itemset com $\mathrm{k}$ elementos é chamado de kitemset.

É chamado suporte, a medida que objetiva avaliar o interesse de uma regra de associação, ele representa a porcentagem de transações de um banco de dados de transações onde a regra se verifica. Dessa forma, o suporte de um itemset é definido como sendo a porcentagem de transações onde este itemset aparece, isto é, onde este itemset está contido, assim, itemsets frequentes são o conjunto de itens que aparecem juntos em pelo menos $\mathrm{x} \%$ das transações, em que o valor de $\mathrm{x}$ é definido pelo suporte.

Em [Agrawal \& Srikant 1994] é apresentada a questão da mineração de regras de associação e para que se possa entendê-la, é preciso definir precisamente o que é uma regra de associação e os diversos conceitos envolvidos.

Diz-se que uma transação $\mathrm{T}$ suporta um itemset I se I $\subseteq \mathrm{T}$. 
VII Congresso Brasileiro de Informática na Educação (CBIE 2018)

Anais do XXIX Simpósio Brasileiro de Informática na Educação (SBIE 2018)

A toda regra de associação $\mathrm{A} \rightarrow \mathrm{B}$ é associado um grau de confiança, denotado por conf $(\mathrm{A} \rightarrow \mathrm{B})$. Este grau de confiança é simplesmente a porcentagem das transações que suportam $B$ dentre todas as transações que suportam $A$, isto é:

$$
\operatorname{conf}(A \rightarrow B)=P(B \mid A)=\frac{\text { número de transações que suportam }(A \cup B)}{\text { número de transações que suportam } A}
$$

Dessa forma, confiança é uma medida objetiva para regras de associação e mede o grau de certeza de uma associação. Em termos estatísticos, trata-se simplesmente da probabilidade condicional $\mathrm{P}(\mathrm{B} \mid \mathrm{A})$, isto é, a porcentagem de transações contendo os itens $\mathrm{B}$ sabendo que os itens $\mathrm{A}$ também ocorreram, ou ainda, probabilidade de ocorrer $\mathrm{B}$ dependendo de A.

Uma regra de associação $r$ é dita interessante se $\operatorname{conf}(r) \geq \alpha$ e $\sup (r) \geq \beta$, em que $\alpha$ e $\beta$ são respectivamente um grau mínimo de confiança e um grau mínimo de suporte especificados pelo usuário.

\subsection{O algoritmo Apriori}

O algoritmo Apriori [Agrawal \& Srikant 1994] resolve o problema da mineração de itemsets frequentes, encontrando esse conjunto de itens usando uma abordagem interativa baseada na geração de candidatos, assim, recebe com entrada um banco de dados de transações $\mathrm{D}$ e um nível mínimo de suporte $\beta$ e fornece como saída todos os itemsets frequentes em $\mathrm{D}$ que satisfaçam $\beta$. Ele possui três fases principais: a fase da geração dos candidatos, a fase da poda dos candidatos e a fase do cálculo do suporte.

\section{Identificação de falhas conceituais}

Os conceitos de itemsets frequentes e regras de associação podem ser aplicados a qualquer domínio, por isso, motivados e fundamentados no que foi apresentado até aqui, foi realizada a instanciação dessas regras para o cenário a seguir.

Considere que após uma aula de biologia, um professor deseje conhecer quão eficaz foi o aprendizado coletivo de sua turma, com a finalidade de assegurar a eficiência de seu trabalho, planejar melhor suas aulas, corrigir tempestivamente desvios e trabalhar conteúdos e conceitos não assimilados por ela. Para isto, resolve aplicar como exercício a construção de um mapa conceitual sobre o tema "Plantas medicinais".

A construção de mapas conceituais [Novak \& Gowin 1984] e [Moreira 2005] é uma estratégia interessante, pois se concentra nos conceitos de um tema em questão e no relacionamento entre eles que o aluno conhece, permitindo assim, não só a explicitação do arcabouço conceitual do aluno como também a medição de resultados a partir da comparação entre conceitos.

Para aferição dos resultados e comparação entre conceitos, o professor precisa de elaborar um mapa conceitual referência. O termo referência associado ao mapa é utilizado pelo fato dele conter conceitos que do ponto de vista do professor devem aparecer nos mapas dos alunos, estabelecendo uma métrica de comparação. Assim, no momento de construção, o professor pode indicar no mapa, dentre todos os conceitos, aqueles que considera mais relevantes. O significado da marcação de conceitos relevantes é importante, pois indica, por meio do juízo do professor, quais são os conceitos indispensáveis de aparecimento nos mapas construídos pelos alunos e quais 
VII Congresso Brasileiro de Informática na Educação (CBIE 2018)

Anais do XXIX Simpósio Brasileiro de Informática na Educação (SBIE 2018)

são periféricos. A Figura 1 apresenta o mapa conceitual referenciado professor sobre o tema plantas medicinais e um exemplo de mapa do aluno.
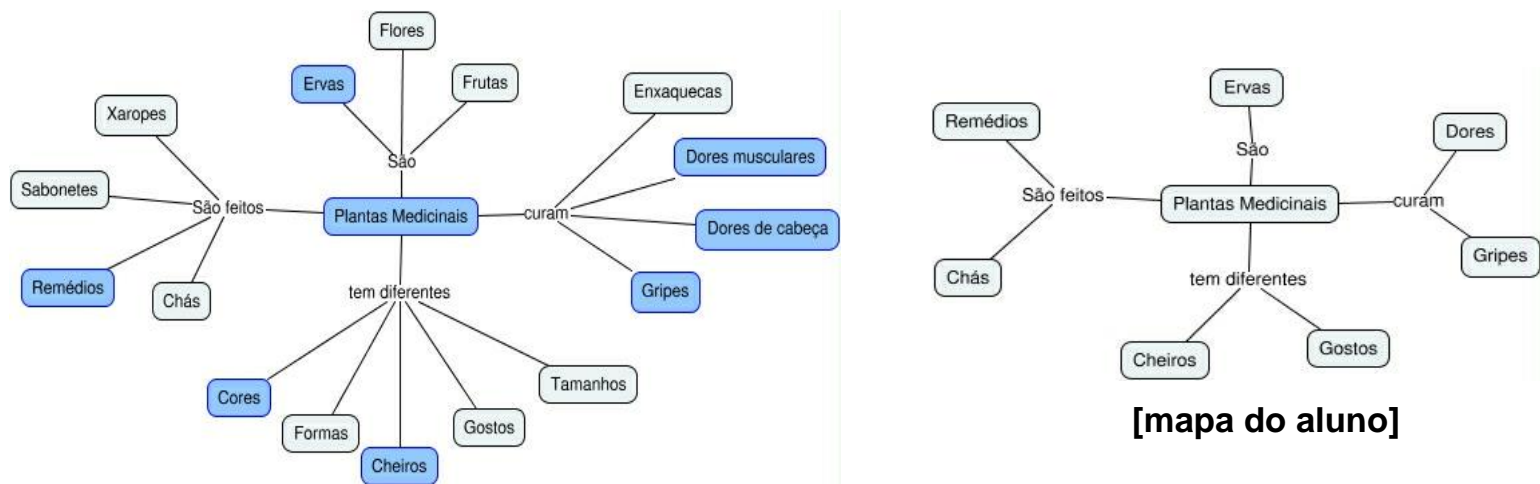

[mapa do aluno]

[mapa referência do professor]

Figura1. Mapa referência do professor e mapa de um aluno sobre o tema plantas medicinais.

Depois de construídos os mapas conceituais dos alunos, o próximo passo é a comparação dos conceitos existentes entre cada mapa e o mapa referência. Essa estratégia para comparação de mapas foi utilizada em outros trabalhos como em [Lamas, Boeres, Cury \& Menezes 2005], entretanto, quando se necessita realizar a multi comparação de mapas de modo a obter um resultado coletivo, essa tarefa se torna demasiadamente complexa, mesmo para espaço amostral de apenas 40 alunos, necessitando de outra abordagem.

Uma questão esperada, devido a aspectos singulares de cada aluno, tais como perspectiva, interesse pelo tema e tempo de estudo sobre ele, é que cada aluno construa seu mapa conceitual diferente do demais, ainda que contenham os mesmos conceitos ou conceitos similares.

O caso de conceitos similares pode ser amenizado realizando-se a sumarização de mapas conceituais [Aguiar, Cury \& Zouaq 2017], que consiste numa revisão de conceitos próximos a fim de, por uma aproximação, escolher um único conceito que represente os demais.

Uma análise detalhada entre os mapas conceituais da Figura 1 permite identificar as divergências entre o mapa conceitual referência e o mapa do aluno. Agora, como estender esse resultado comparativo para uma abordagem coletiva ou de forma mais específica, como é possível verificar com suporte e confiança suficientes quais conceitos foram assimilados pela turma?

A resposta a essa pergunta é importante porque fornece ao professor o subsídio para que possa trabalhar o conjunto ausente de conceitos, ou seja, aqueles que de forma coletiva não foram assimilados pela turma e por isso não constam de forma geral nos mapas conceituais, além de permitir verificar o surgimento de conceitos que foram apontados pelos alunos e não foram considerados no mapa referência elaborado pelo professor, entretanto, emergiram como parte do conhecimento coletivo.

Com o objetivo de responder à questão anterior, foram aplicados os conceitos de suporte e confiança no contexto de itemsets frequentes e regras de associação já apresentados e, de forma mais específica, é enunciada a definição a seguir. 
VII Congresso Brasileiro de Informática na Educação (CBIE 2018)

Anais do XXIX Simpósio Brasileiro de Informática na Educação (SBIE 2018)

Definição 1: Dado um banco de dados de transações D compostas por itemsets (em que cada itemset pertencente a uma transação é constituído do conjunto de conceitos de um mapa conceitual), um nível mínimo de confiança $\alpha$ e um nível mínimo de suporte $\beta$, então, os itemsets frequentes e as regras de associação interessantes com relação a $\mathrm{D}, \alpha$ e $\beta$ representam, para esses parâmetros, os conceitos associados, assimilados e aprendidos coletivamente.

O significado da definição 1 traduz um relacionamento de associação semântica entre os conceitos, uma vez que, em termos das regras de associação, exprimem que sempre que um conceito é lembrado outro também é, estando assim relacionados por proximidade semântica com valores de suporte e confiança.

Para exemplificar a definição 1, considere a Figura 2 que contém em (i) $a$ relação de conceitos do mapa referência, incluindo a indicação se um conceito é ou não relevante.

\begin{tabular}{|c|c|c|c|c|c|}
\hline I & Id & Conceito (item) & Relevante & Transação & Itemsets de conceitos \\
\hline I & 1 & Plantas Medicinais & Sim & 1 & $\{1,2,3,4,5,7,8,9,11,13,14,17\}$ \\
\hline ! & 2 & Sabonetes & Não & 2 & $\{1,3,5,7,8,10,11,13,14\}$ \\
\hline i & 3 & Xaropes & Não & 3 & $\{1,2,3,4,5,8,9,11,13,14,17\}$ \\
\hline I & 4 & Remédios & Sim & 4 & $\{1,3,5,9,11,17\}$ \\
\hline I & 5 & Chás & Não & 5 & $\{1,2,3,4,5,7,8,9,11,13,14,17\}$ \\
\hline I & 6 & Frutas & Não & 6 & $\{1,2,3,5,8,9,13,14,17\}$ \\
\hline i & 7 & Flores & Não & 7 & $\{1,2,3,5,8,9,11,13,14,17\}$ \\
\hline I & 8 & Ervas & Sim & 8 & $\{1,2,3,5,8,9,11,14,17\}$ \\
\hline ! & 9 & Cores & Sim & 9 & $\{1,3,5,8,9,13,14,17\}$ \\
\hline i & 10 & Formas & Não & 10 & $\{1,2,3,5,8,11,13,14,17\}$ \\
\hline I & 11 & Tamanhos & Não & \multirow{7}{*}{\multicolumn{2}{|c|}{ (ii) }} \\
\hline I & 12 & Gostos & Não & & \\
\hline 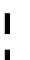 & 13 & Cheiros & Sim & & \\
\hline i & 14 & Gripes & Sim & & \\
\hline 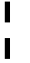 & 15 & Dores Musculares & Sim & & \\
\hline 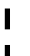 & 16 & Dores de Cabeça & Sim & & \\
\hline I & 17 & Enxaquecas & Não & & \\
\hline
\end{tabular}

Figura 2. Representação de cada conceito do mapa conceitual referência em (i) e os itemsets dos mapas dos alunos em (ii)

Nesse ponto, precisa-se elicitar qual é o conjunto de k-itemsets produzidos a partir dos mapas conceituais dos alunos, para isso, são apresentados como exemplo, alguns desses itemsets na Figura 2 (ii). Cada transação representa um mapa conceitual 
VII Congresso Brasileiro de Informática na Educação (CBIE 2018)

Anais do XXIX Simpósio Brasileiro de Informática na Educação (SBIE 2018)

produzido por um aluno num evento de aprendizagem e cada itemset, o conjunto de conceitos desse mapa, referenciados na Figura $2(i)$.

Uma vez obtidos os itemsets, suponha que o professor decida conhecer qual a lista de conceitos (itemsets frequentes) aparecem em pelo menos $80 \%$ de todos os mapas conceituais registrados, contendo $100 \%$ de confiança em suas regras de associação (neste caso, com suporte $\beta=0,8$ e confiança $\alpha=1$ ). Para isso, procede-se a execução do algoritmo Apriori e a seleção de itemsets e regras gerados a fimm de atender aos parâmetros $\beta$ e $\alpha$.

A semântica por trás da seleção de itemsets com esses parâmetros é que pode-se, a partir da lista retornada, verificar os itemsets frequentes e a regra de associação $r$ que possa ser considerada interessante e relevante com $\operatorname{conf}(\mathrm{r}) \geq \alpha$ e $\sup (\mathrm{r}) \geq \beta$, em que $\alpha$ e $\beta$ são respectivamente um grau mínimo de confiança e um grau mínimo de suporte especificados pelo usuário, no nosso exemplo, $\alpha=1$ e $\beta=0,8$.

Adicionalmente, pode-se considerar que o relacionamento entre os conceitos de um mapa conceitual que constituem um itemset e pertencem a uma determinada regra de associação, possuem, para o aluno, um valor de ligação semântica, análoga ao relacionamento existente entre os objetos de aprendizagens acessados conjuntamente num ambiente virtual ou ao conjunto de itens escolhidos numa compra no supermercado, dados no contexto das regras de associação, pela regra do lado da mão esquerda e lado da mão direita.

Em termos gerais, isso significa que, coletivamente, foram os principais conceitos percebidos e/ou lembrados e associados num evento de aprendizado, porque juntos, não só satisfazem e completam um contexto semântico pelo seu encadeamento, como também permitem uma ligação que delimitam uma região conceitual semântica, coincidentes com o arcabouço conceitual coletivo, como mostra a figura 3 a seguir.

A Figura 3 exemplifica o conceito de região conceitual semântica dito anteriormente, em que cada arcabouço conceitual é delimitado pelas linhas vermelhas. Assim, mesmo que haja ligações entre eles por meio de conceitos pertencentes a cada região semântica, como ocorre entre os conceitos "Plantas" e "Plantas Medicinais", cada região pode ser bem definida em termos do contexto e significado dos relacionamentos entre seus conceitos. Para o nosso caso, os dois contextos são Plantas Medicinais e Medicamentos Naturais.

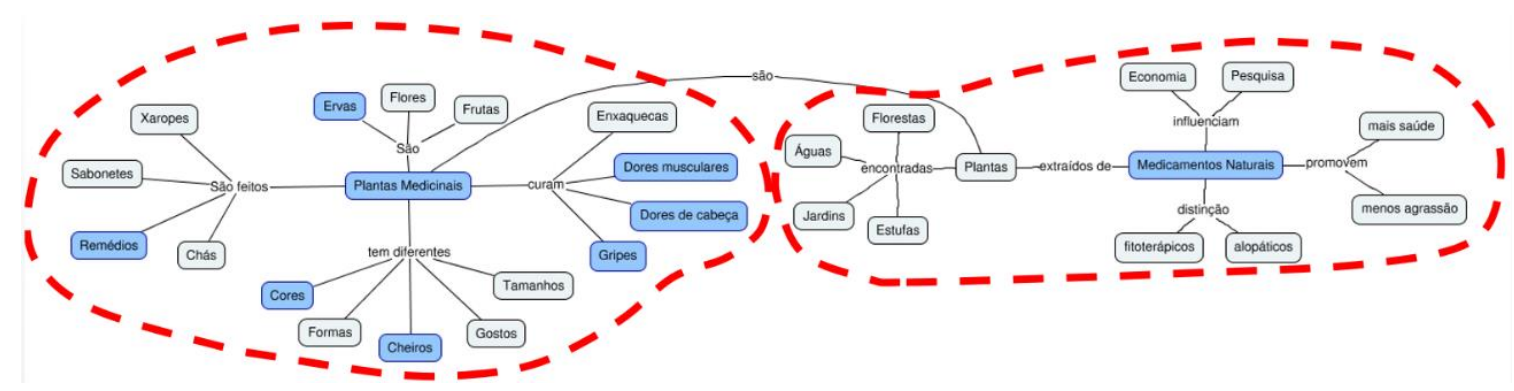

Figura 3. Regiões semânticas delimitada pelo contexto.

\subsection{Variando o suporte e a confiança}

Para o exemplo apresentado na Figura 2 (ii), o resultado para o valor de suporte $\beta=1 \mathrm{e}$ confiança $\alpha=0,8$ retorna a seguinte lista de conceitos: 
VII Congresso Brasileiro de Informática na Educação (CBIE 2018)

Anais do XXIX Simpósio Brasileiro de Informática na Educação (SBIE 2018)

$$
\mathrm{R}=\{1,3,5\}=\{\text { Plantas Medicinais, Xaropes, Chás }\}
$$

Quando resultados como o anterior aparecem, tendem a ser pouco relevantes, uma vez que apresentam um conjunto pequeno de itens comparando-se com total presente no banco D (Figura 2 (i)), dessa forma, é necessário fazer uma variação dos parâmetros de suporte e confiança até que se encontre o resultado desejado.

Uma perspectiva interessante é a visualização dos k-itemsets e suas regras quando se variam os valores de suporte e confiança. A Tabela 1 exibe um exemplo de kitemsets resultado possíveis. Observe que para mesmos valores de $\beta$ são gerados diferentes k-itemsets resultado, por isso, utilizou-se como critério de seleção aqueles que possuem maior tamanho $\mathrm{k}$ e que contenham maior quantidade de conceitos marcados como relevantes pelo professor. $\mathrm{Na}$ tabela 1 abaixo são indicados esses elementos com marcação em negrito e com asterisco.

Tabela 1. k-itemsets resultado a partir da variação do suporte

\begin{tabular}{|c|c|c|}
\hline Suporte $(\beta)$ & Confiança ( $\alpha)$ & k-itemsets resultado \\
\hline 1 & 0,8 & $\{1,3,5\}^{*}$ \\
\hline 0,8 & 0,8 & $\{1,3,5,8,13\},\{1,3,5,8,14\},\{1,3,5,8,14,17\},\{\mathbf{1}, \mathbf{3}, \mathbf{5}, \mathbf{8}, \mathbf{1 3}, \mathbf{1 4}\}^{*}$ \\
\hline 0,6 & 0,8 & $\begin{array}{c}\{1,2,3,5,8,9,14,17\},\{1,2,3,5,8,11,14,17\},\{1,2,3,5,8,13,14,17\}, \\
\{\mathbf{1 , 3 , 5 , 8 , 9 , 1 3 , 1 4 , 1 7}\}^{*}\end{array}$ \\
\hline 0,4 & 0,8 & $\{1,2,3,5,8,9,11,13,14,17\}^{*}$ \\
\hline
\end{tabular}

Uma implicação direta da variação paramétrica dos valores de suporte e confiança é a obtenção de valores mínimos que satisfaçam o conjunto de requisitos de avaliação de aprendizagem desejado pelo professor.

Assim, caso o professor tenha em mente que seja satisfatório que a turma tenha uma compreensão de conceitos com suporte de $80 \%$ e regras de associação com confiança de $80 \%$ ele pode, com esses valores de parâmetros, obter não só o conjunto de conceitos assimilados como também o conjunto de conceitos complementares que são, como implicação derivada desse resultado, o conjunto que representa os conceitos ausentes ou que não satisfizeram os parâmetros de suporte e confiança decorrentes dos requisitos de avaliação e por isso, devem ser trabalhados posteriormente de modo a preencher essa lacuna de conhecimento.

Nesse exemplo, percebe-se que mesmo que os conceitos tenham um valor considerável de confiança (80\%), apenas quando um valor de suporte de $40 \%$ é atingido é que se obtêm resultados relevantes, ou seja, a cobertura de 58,8\% (10/17) da quantidade de conceitos do mapa original. Quando o suporte é de $80 \%(\beta=0,8)$ é conseguida cobertura de somente $35,3 \%(6 / 17)$ da quantidade de conceitos do mapa, mesmo com a confiança de $80 \%$ para algumas regras de associação.

Essa informação é importante porque explicita preliminarmente duas coisas:

A primeira e mais evidente é que é necessário trabalhar melhor alguns conceitos pois resultados relevantes começam a aparecer apenas para valores muito baixos de suporte.

A segunda é a influência da confiança para o resultado final, apontando que o seu valor, que é a probabilidade de ocorrência de um item quando outro ocorre, diz respeito às regras, assim, não é suficiente para sozinha, gerar resultados relevantes. 
VII Congresso Brasileiro de Informática na Educação (CBIE 2018)

Anais do XXIX Simpósio Brasileiro de Informática na Educação (SBIE 2018)

Na figura 4, é apresentado o resultado da Tabela 1 para variações dos valores de suporte.

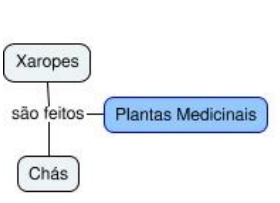

$[\beta=1]$

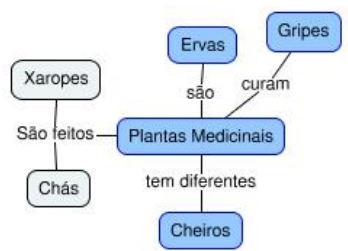

$[\beta \geq 0,8]$

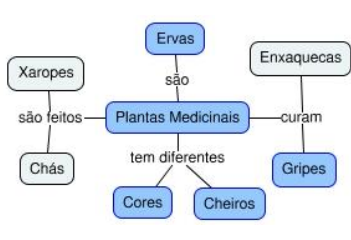

$[\beta \geq 0,6]$

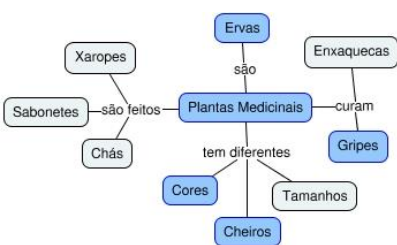

$[\beta \geq 0,4]$

Figura 4. Mapas conceituais gerados a partir da variação do suporte

\subsection{A escolha de conceitos ausentes}

A principal contribuição ao realizar a análise dos itemsets frequentes resultado e das regras de associação em mapas conceituais é a verificação dos conceitos aprendidos pelo grupo de alunos e aqueles cuja ausência merecem atenção para serem posteriormente reapresentados sobre outra perspectiva ou com outra estratégia de ensino para que possam ser melhores assimilados coletivamente.

Tão importante quanto o conhecimento dos conceitos assimilados é a apresentação do conjunto de conceitos ausentes ou aqueles que não satisfizeram os critérios de suporte e confiança, pois devem ser retrabalhados de modo a obedecer a critérios que otimizem o processo cognitivo. Assim, questões como a metodologia, abordagem pedagógica e a ordem de precedência com que são apresentados devem ser pensados para que seja potencializado o aprendizado do aluno.

Sobre essa perspectiva e como decorrência direta das regras de associação, uma abordagem a ser executada é a apresentação de conceitos de acordo com os valores progressivos de suporte $(\beta)$. Por exemplo:

Considere que o professor ache satisfatório, valores iniciais de suporte $\beta=0,6 \mathrm{e}$ confiança $\alpha=0,8$ para que a partir daí possa trabalhar os conceitos restantes, não presentes para esses parâmetros de $\beta$ e $\alpha$.

A pergunta a ser respondida é: Quais os próximos conceitos devem ser apresentados de modo a otimizar o aprendizado? Ou seja, em vez de apresentar os elementos restantes de forma aleatória, concentramo-nos em escolhê-los meticulosamente, de modo a valorizar os conceitos que são considerados mais relevantes e que estão associados, utilizando para isso as regras de associação elicitadas quando da execução do algoritmo Apriori.

Atribuindo-se níveis, apenas para questões de referência, para os conjuntos de itemsets da Tabela 1, em que cada nível é delimitado pelos diferentes valores de $\beta$ e $\alpha$, é obtida a Tabela 2. Essa tabela apresenta os itemsets escolhidos a partir do critério de relevância e tamanho apresentado na seção 3.1, os itemsets diferença entre os k-itemsets resultado de níveis imediatamente subsequentes, regras de associação relevantes geradas para os elementos do conjunto diferença e os conjuntos complementares de conceitos para os valores de $\beta$ e $\alpha$ selecionados. 
VII Congresso Brasileiro de Informática na Educação (CBIE 2018)

Anais do XXIX Simpósio Brasileiro de Informática na Educação (SBIE 2018)

Tabela 2.Classificação de itemsets de acordo com níveis

\begin{tabular}{|c|c|c|c|c|c|c|}
\hline Nível & $(\boldsymbol{\beta})$ & $(\boldsymbol{\alpha})$ & k-itemset Resultado & $\begin{array}{c}\text { Conjunto } \\
\text { Diferença }\end{array}$ & $\begin{array}{c}\text { Regras de } \\
\text { Associação }\end{array}$ & $\begin{array}{c}\text { Conjunto } \\
\text { Complementar }\end{array}$ \\
\hline 1 & 1 & 0,8 & $\{1,3,5\}$ & $\{8,13,14\}$ & $\begin{array}{c}\{8\}=>\{14\}=100 \% \\
\{8\}=>\{13\}=88,9 \%\end{array}$ & $\begin{array}{c}\{2,4,6,7,8,9,10,11,12 \\
, 13,14,15,16,17\}\end{array}$ \\
\hline 2 & 0,8 & 0,8 & $\{1,3,5,8,13,14\}$ & $\{9,17\}$ & $\{9\}=>\{17\}=100 \%$ & $\begin{array}{c}\{2,4,6,7,9,10,11,12,1 \\
5,16\}\end{array}$ \\
\hline 3 & 0,6 & 0,8 & $\{1,3,5,8,9,13,14,17\}$ & $\{2,11\}$ & $\{2\}=>\{11\}=87,5 \%$ & $\{2,4,6,7,10,11,12,15$, \\
$16\}$
\end{tabular}

Considerando que o itemset resultado para um nível que possua maior valor de suporte, é também o que contém os elementos melhores assimilados pela turma, a diferença entre cada itemset resultado de níveis imediatamente subsequentes são os elementos não compreendidos entre esses níveis.

Por exemplo, para o nível 1 , o itemset resultado $\{1,3,5\}$ indica que esses foram os elementos melhor assimilados para o valor de $\beta=1$ e o itemset diferença $\{8,13,14\}$ exprime a diferença entre os elementos dos itemsets resultado de níveis 1 e 2 . Assim, os elementos $\{8\},\{13\}$ e $\{14\}$ são os elementos pertencentes ao nível 2 que não satisfazem os critérios de $\beta$ para o nível 1, pois possuem valores inferiores de suporte. Dessa forma, caso o professor deseje trabalhar a lacuna de conhecimento entre esses níveis (nível 1 e 2), deve primeiro apresentar, prioritariamente, os conceitos divergentes entre eles antes de concentrar esforços no itemset complementar de mesmo nível, neste caso, $\{2,4,6,7,8,9,10,11,12,13,14,15,16,17\}$ uma vez que são mais próximos em termos de valores de suporte. Complementarmente, perceba que o conjunto diferença é também um subconjunto do conjunto complementar de elementos, por exemplo, o conjunto diferença de nível $1\{8,13,14\} \subseteq\{2,4,6,7,8,9,10,11,12,13,14,15,16,17\}$, seu conjunto complementar.

Observe que não há prioridade entre os conceitos $\{8\}$ - "Ervas", $\{13\}-$ "Cheiros" e $\{14\}$ - "Gripes", uma vez que ambos foram marcados como relevantes pelo professor (Figura $2(i)$ ), entretanto, quando se segue para o nível subsequente (nível 3), note que o conceito $\{9\}$ - "Cores" é prioritário sobre $\{17\}$ - "Enxaquecas", o outro do conjunto diferença $\{9,17\}=$ CCores, Enxaquecas $\}$ de mesmo nível, como mostra a Figura 5. Isso significa que quando o professor for reapresentar os conceitos, deve primeiro apresentar o conceito "Cores" antes de "Enxaquecas".
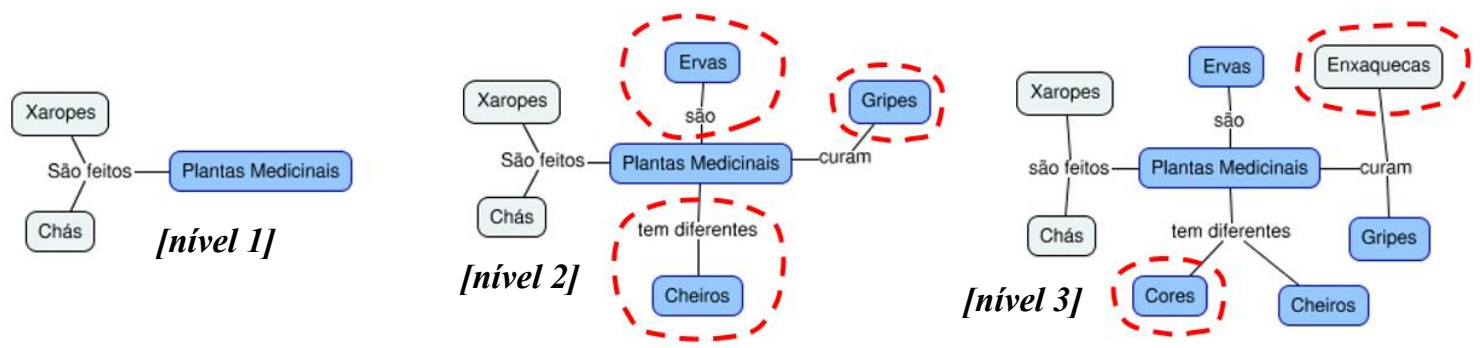

Figura 5. Prioridade de apresentação dos conceitos com a mudança de níveis.

Depois que os conceitos prioritários de nível foram reapresentados, pode-se utilizar as regras de associação para os demais conceitos de nível, assim, para cada item, apresentar os que possuem maior percentual de confiança $(\alpha)$ (Tabela 2), ou seguir para os conceitos prioritários de próximo nível. Essa estratégia objetiva trabalhar primeiro os 
VII Congresso Brasileiro de Informática na Educação (CBIE 2018)

Anais do XXIX Simpósio Brasileiro de Informática na Educação (SBIE 2018)

conceitos pertencentes a itemsets e regras de associação de níveis próximos a fim de que, uma vez cobrindo os conceitos que tenham menores valores de suporte e confiança do nível imediatamente inferior, eles possam fazer parte, nas próximas transações, dos itemsets com maiores valores de suporte e confiança pertencentes ao nível imediatamente superior, indicando assim, uma maturação do aprendizado da turma.

\section{Considerações finais}

A partir do uso da técnica de regras de associação aplicada em mapas conceituais propusemos uma abordagem para identificar falhas conceituais em estudantes, apontando, não só os conceitos não compreendidos por uma turma de aprendizes num evento de aprendizagem, mas também a sequência mais adequada em que devem ser apresentados de modo a permitir que se possa nivelar, de forma otimizada, o conhecimento coletivo da turma a um mesmo patamar. Essa técnica é a parte preliminar de um arcabouço de ensino e sua contribuição é o fornecimento de um valioso conhecimento necessário para orientar professores na tarefa de ensino de forma mais assertiva, considerando aquilo que já se conhece da turma a partir de um primeiro contato. Esse arcabouço proposto foi verificado em ambiente de laboratório e os resultados nos mostram até o momento, seu potencial na assistência adaptada, quer seja individuada ou de grupos, além disso, os próximos testes estão previstos com turmas reais. Desdobramentos deste trabalho relacionam-se à sua aplicação em contextos de ensino intermediados por sistemas tutores inteligentes ou outros agentes de software.

\section{Referências}

Agrawal, R., \&Srikant, R. (1994). Fast Algorithms for Mining Association Rules. Proceedings of the 20th VLDB Conference. Santiago, Chile.

Aguiar, C. Z., Cury, D., \&Zouaq, A. (2017). Mineração de Mapas Conceituais para Sumarização de Textos. VI Congresso Brasileiro de Informática na Educação - SBIE.

Amo, S. d. (2004). Técnicas de Mineração de Dados. XXIV Congresso da Sociedade Brasileira de Computação. Salvador.

Cury, D., Perin, W. A., \& Jr., P. S. (2014). CMPAAS - A Plataform of Services for Construction and handling of Concept Maps. Proceedings of Sixth Internacional Conference of Concept Maps, (pp. 108-115).

Flôres, M. L., Tarouco, L. M., \&Reategui, E. B. (2009). Orientações para o sequenciamento das instruções em um objeto de aprendizagem. RENOTE - Revista Novas Tecnologias na Educação, v. 7, n. 1.

Han, J., Kamber, M., \&Pei, J. (2011). Data Mining Concepts and Techniques. Elsevier.

Lamas, F., Boeres, C., Cury, D., \& Menezes, C. S. (2005). Comparando mapas conceituais utilizando correspondência de grafos. Simpósio Brasileiro De Informática Na Educação - SBIE, (pp. 24-27).

Moreira, M. A. (2005). Mapas Conceituais e Aprendizagem Significativa. Revista Chilena de Educação Científica, 38-44.

Novak, J. D., \&Gowin, D. B. (1984). Learning How to Learn. Cambridge University Press. 